\title{
A novel signal processing method for friction and sliding wear
}

Rajini Nagarajan ${ }^{\text {a, *, Karthikeyan Subramanian a }}$, Saravana Sankar Subramaniam ${ }^{\text {b }}$

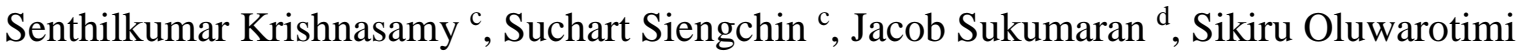
Ismail $^{\text {e }}$, Faruq Mohammad ${ }^{\mathrm{f}}$, Hamad A. Al-Lohedan ${ }^{\mathrm{f}}$

${ }^{a}$ Centre for Composite Materials, Department of Mechanical Engineering, Kalasalingam Academy of Research and Education, Krishnankoil, 626126, Tamilnadu, India.

${ }^{\mathrm{b}}$ Department of Mechanical and Process Engineering, Automotive Safety and Assessment Engineering (ASAE), The Sirindhorn International Thai-German Graduate School of Engineering (TGGS), King Mongkut's University of Technology North Bangkok, 1518

Wongsawang Road, Bangsue, Bangkok 10800, Thailand.

${ }^{c}$ Department of Materials and Production Engineering, The Sirindhorn International ThaiGerman Graduate School of Engineering (TGGS), King Mongkut's University of Technology

North Bangkok, 1518 Wongsawang Road, Bangsue, Bangkok 10800, Thailand.

${ }^{\mathrm{d}}$ Department of Electrical Energy, Metals, Mechanical Construction and Systems, Ghent University, Technologiepark, Zwijnaarde 903, Ghent, Belgium.

${ }^{\mathrm{e}}$ Manufacturing, Materials, Biomedical and Civil Division, School of Engineering and Computer Science, University of Hertfordshire, Hatfield, Hertfordshire AL10 9AB, England, United Kingdom.

${ }^{\mathrm{f}}$ Department of Chemistry, College of Science, King Saud University, P.O. Box 2455, Riyadh, Kingdom of Saudi Arabia 11451.

*Corresponding authors:

Rajini Nagarajan, E-mail: rajiniklu@ gmail.com, Tel.: +919942139392; Fax: +914563289042. Suchart Siengchin, Email: suchart.s.pe @ tggs-bangkok.org

\begin{abstract}
This current study proposed a new computationally efficient and comparatively accurate algorithm for calculating both static and dynamic coefficients of friction from high-frequency data. Its scope embraced an application in a real-time friction-based system, such as active braking safety systems in automobile industries. The signal sources were from a heavy duty reciprocating dry sliding wear test platform, focused on experimental data related to friction
\end{abstract}


induced by stick-slip phenomena. The test specimen was a polytetrafluoroethylene (PTFE) coated basalt/vinyl ester composite material, tested at a large scale. The algorithm was primarily aimed to provide scalability for processing a significantly large tribological data in a real-time. Beside a computational efficiency, the proposed method adopted to evaluate both static and dynamic coefficients of friction using the statistical approach exhibited a greater accuracy and reliability when compared with the extant models. The result showed that the proposed method reduced the computation time of processing, and reduced the variation of the absolute value of both static and dynamic frictions. Although, the variation of dynamic friction was later increased at a particular threshold, based on the test duration.

Keywords: Friction; data processing; stick-slip; basalt/vinyl ester composite.

\section{Introduction}

In engineering applications, stick-slip phenomena are non-beneficial in a tribological process, due to the vibration and audible noise [1,2]. The understanding of the stick-slip phenomenon is critical, especially in many applications, such as brakes and plain bearings. This occurrence predominantly occurs in a contact between two sliding surfaces. The stick-slip occurs at the time when static friction exceeds the dynamic friction and friction coefficient reduces suddenly $[3,4]$. Hence, it is essential to study the relationship between different tribological signals for a better understanding of a material, especially about its stick-slip behavior. In this regards, many attempts have been made to understand the stick-slip occurrences, with the help of experimentation, mathematical and theoretical modellings. The theoretical and experimental analyses of stick-slip, using rubber materials were studied in the early 1970s [5]. Recently, Real et al. [6] developed a new test rig to analyze stick-slip cycles; thereby, the stick-slip signals were studied in a detailed manner with hysteric cycles. Dong et al. [7] studied the deformation behavior and stick-slip phenomenon of polymer materials under water lubrication, heavy load and lower speed conditions. It was evident from their research that the velocity played a major role in the deformation and stick-slip phenomenon. Similar work has been carried out on 4 different polymer groups, sliding against the cast-iron counter surface. An attempt was also made to study the stick-slip phenomena using high-speed in-situ imaging and force measurement in the polymer materials [8]. The different modes of stick-slip was observed in a low-velocity sliding. 
The uniform waves were visible along with a stick. The slip pulses were also described clearly. However, the processing of high-frequency signals on measuring the performance of tribological outcome or parameters still required more considerable attention, in terms of their computational processing and evaluation.

Moreover, signal processing in a wear application has started increasing, especially towards defect detection in machining application [9]. There were methods for processing a signal in the past decades. They were not suitable for handling multi-sensor architecture and its related method of data analysis. The use of artificial neural networks and other computational programming methods to process a huge data also have their limitations. The friction-induced noise/vibration and stick-slip are often a non-linear function [10,11]. Therefore, they require a careful and accurate recording of frictional forces to reduce the squeal noise signals. Thereby, a high-frequency data acquisition with multiple sensors is a promising alternative to visualize the stick-slip trend. The need for improvement in algorithms is essential to avoid errors and obtain an efficient data processing.

In additions, many researchers had used signal processing techniques to analyze the wear parameters. For instance, Bhattacharyya et al. [12] analyzed the tool force in a real-time, using various signal processing techniques, such as a linear filtering, time domain and wavelet transformation. All these methods have been used to achieve a relative high accuracy and reliability for wear estimation. These approaches adopted the statistical model to ensure the consistency of the signal processing outcomes. They showed better performance in terms of accuracy and reliability than the earlier methods of signal processing. Kong et al. [13] developed a novel wear assessment technique for a real-time and accurate monitoring of various tool wear parameters. The Gaussian process-regression method showed a better performance in data processing than the artificial neural network. The removal of noise from the signal and further processing were made easier, using the Gaussian method. This techniqueh helped to monitor the wear accurately. Neis et al. [14] studied the grown creep parameters caused by stick-slip using a tribometer. The correlation between the accelerometer signal and the stick-slip parameter were analyzed, using 3 different friction materials. The results showed a high correlation between those parameters. Similarly, they correlated the brake creep growth and stick-slip at a low velocity, after taken measurements with an accelerometer that was placed on the brake calipers. 
A fast Fourier transform spectrum data during acceleration were captured in both front and rear wheels. The data were used to validate their experimental results by comparison [15]. Masotti et al. [16] tested non-asbestos organic brake material using a tribometer for the stick-slip study, and the surface morphology of the same material were reported. An algorithm was developed on image segmentation technique and it was used to process the image taken from the test and calculate the real contact area of the material. Eventually, the correlation between the applied torque and the stick-slip were studied.

The need for a multi-sensor data at a higher frequency for extraction of accurate tribological data has been considered, and later it was invented for processing a large data sets. Nevertheless, the advancement in cloud-based servers opened a new door to handle big data in a cost-effective manner. Instead of investing a huge amount of money in processor and software, the cloud-based server provides a platform for researchers to analyze a very huge amount of data in a fast speed. It becomes a user-friendly environment for the individual user and the industrial members to work on the server without the fear of data loss. Also, cloud servers allow users to choose the speed and storage capacity, depends on the application and need. More also, the stickslip phenomenon is measured in a various engineering applications, such as automobile, environmental studies and aviation, to mention but a few. Every sector tries to capture stick-slip using active and passive methods. One of the passive methods is made using wireless environmental sensor network. The sampling rate used in this method is very high, similar to the use of servers hosted in the Amazon cloud web service to process the data acquired using sensors [17]. Dimla [18] reviewed the use of multi-sensor to monitor the wear rate of the tool and its various methods. The cutting force and the vibrations were measured using sensors, and all the data were analyzed to predict the tool wear. In sensor fusion, where many sensors were connected to measure a particular parameter in an attempt to eradicate noise and processing the same gives the desired output value with more precision. Application of multi-sensor signal to predict the wear and stick-slip was commonly used before to calculate the wear of work pieces and tools. Under different machining operations: drilling, turning, broaching and milling operations, the use of sensors to calculate wear parameters are now common. Boud and Gindy [19] monitored the workpiece and tool using multi-sensor system to obtain cutting force, displacement and pressure signals. Acoustic emission signals were effectively used to detect the noise and other irregularities during machining. Additionally, Chung and Geddam [20] acquired 
both acoustic and force signals using a multi-sensor system. These signals were monitored online, and signal processing technique of fast Fourier transform (FFT) frequency averaging was adapted to reduce the noise. Segreto et al. [21] used a sensor fusion method to monitor the tool wear when turning the nickel alloy and applied the neural network pattern recognition method. This method combined all the sensor data to measure the tool wear precisely. One of the unexplored applications of stick-slip occurs in a large scale composite bearing. Furthermore, an ease of processing methods with moderate hardware system can make the researchers more comfortable to analyze a high-frequency data. Accordingly, the high-frequency data obtained from the stick-slip phenomena in a large scale composite bearings can be analyzed through a cloud computing concepts.

Hence, the main aim of this paper addresses the shortcomings of existing processing method used for the static and dynamic friction measurements, as highlighted by Bonny et al. [23]. They accounted for the average of peak values and therefore, resulted to elimination of small peaks, which were identified when processing with aid of formula. However, the current research aims to capture the stick-slip phenomenon, precisely with a multi-sensor architecture. A high-frequency data was acquired for capturing the stick-slip signals. Therefore, the friction data were processed and analyzed from the stick-slip signals obtained from the sliding contact under a high loading condition in this study. A cloud server was utilized to perform the task with the aid of a novel algorithm to calculate the static and dynamic friction coefficients.

\section{Experimental details}

\subsection{Materials and methods}

The composite material (Basalt/PTFE-polyester) used in this current research was developed in-house, more information about the processing methodologies can be found from our earlier work [19]. In this composite, a $50 \mathrm{wt} \%$ of basalt fiber was used as primary reinforcement due to its better mechanical properties [22]. The top layer of the composite contact surface was blended with PTFE to improve the tribological performance. The schematic of the layered composite used is shown in Fig. 1.

\subsection{Tribological setup and conditions}


Dry sliding wear tests on PTFE (10 wt\%) blended basalt fiber/vinyl ester (layered) hybrid composites were performed, using a medium scale flat (MSF) testing machine, available at the Laboratory Soete, Ghent University, Belgium. Experiments were performed on a flat-on-flat contact, using tailor-made composite tested against steel counter face (100 Cr6 steel). The counter plate was machined to $200 \times 80 \times 20 \mathrm{~mm}$ from round bars and subsequently, grounded to surface roughness $\left(R_{a}\right)$ of $-0.2 \mu \mathrm{m}$. Fig. 2 shows the schematic diagram of the experimental set up, with the wear samples on both sides of the sample holders. During testing, various signals such as wear, static and dynamic friction forces, temperature, vibration from triaxial accelerometer and sound were recorded online. All tests were conducted at $25{ }^{\circ} \mathrm{C}$ ambient temperature. Three identical tests were performed to study the repeatability of the stick-slip phenomena. The test conditions are tabulated in Table 1.

\subsection{Method of allocation of computing resource for digital signal processing algorithms}

Data accusation was performed at a sampling rate of $20 \mathrm{kHz}$. Therefore, for every cycle, the total number of data points were 80,000 $(4 \times 20,000 \mathrm{~Hz})$. The periodic time was 4 seconds. The experiment was conducted for about 3 hours and incorporated multiple sensors; therefore, each sensor's corresponding total number of data points for each test was $216 \times 10^{6}(3 \times 60 \times 60$ x $20000 \mathrm{~Hz}$ ). The acquired data for all channels were extended double precision floating point numbers. Therefore, each data point of each channel occupied 128 bit (16 bytes) of storage. During data accusation both hardware and software components created a minimum of 3 channels (time, raw data and data index) for each sensor used. For instance, the accelerometer has a time, x-data, y-data, z-data, and a common index channels. Therefore, the minimum total storage space for each signal was approximately10 Gigabytes: 16-bytes x 3 (no of channels) $\mathrm{x}$ $\left.216 \times 10^{6}\right)$. The compressed file format used to store the measurement data during the experiment was D7D (the DAQ software-DEWE soft-native file format), whereas an uncompressed file format used for post-processing and further analysis of the measurement data was technical data management streaming (TDMS), national instruments (NI) file format. The raw data channels (voltage values) were converted to the corresponding engineering units (EU) by the required scaling factor before post-processing. 
During data accusation, the data passed from the external hardware (DAQ card) to the system (workstation computer) with the help of data accusation software (DEWEsoft). Some simple arithmetic calculations, such as scaling factor multiplications, offset corrections were performed with the help of cache memory (static random accessible memory) alongside the accusation process, therefore, eliminating the need for a post-processing stage. The buffer memory was set to 1 Gigabyte of system memory, known as a dynamic random accessible memory (DRAM). Therefore, whenever the buffer was full, the data in the DRAM was moved to the secondary storage of the system's hard disk drive (HDD). This process effectively removed the requirement of more physical memory in the workstation computer. Although, the secondary storage needed to be fast enough to move the data before the next buffer and prevented data loss. In this work, the physical memory requirement for the workstation was about 1 gigabyte. For the post-processing as well as running analysis algorithm especially focus on real-time implementation, the entire signal data needed to be in the RAM system was approximately 10 Gigabytes. Also, the operating system (OS) and the programming environment which hosted the methods required to be 64-bit memory. A 32-bit environment is limited to only 4 Gigabytes memory. For this method of analysis, a total of 24 Gigabyte RAM was needed, because the system used about 12 Gigabyte RAM. This included the OS and other preliminary requirements for the input signal and another 12 Gigabyte for the output signal after calculation. The only drawback of this method was that it required the same amount of RAM, as that of the signal. However, the real-time implementation required less memory for the finalized algorithms, based on the scalability of the application. A high specification system was used through cloud computing. The cloud system (cloud post-processing workstation computer) used a Window server 2016 64-bit OS, 32 Gigabyte DDR4 ECC RAM, Intel Xeon processor (8 cores with allcore boost frequency of about $2.6 \mathrm{GHz}$ ). The acquired signal data were transferred to the cloud computing system from the DAQ workstation computer.

\section{Results and discussion}

\subsection{Drawbacks of calculating friction using existing methods}

The experimental analysis in this work involved reciprocating dry sliding wear test platform, both static and dynamic friction coefficients were studied from the behavior of the friction force, concerning time and machine's dynamic characteristics. Fig. 4 represents the 
general friction curve pattern for the dry sliding reciprocating wear system, where the multiple time zones correspond to representing different characteristics of the friction behavior was also indicated. Fig. 4 depicts one complete cycle consists of $t_{1}, t_{2}$ and $t_{3}$.

Where, $t_{1}$ is the time frame corresponding to static friction data of test cycle, $t_{2}$ represents the positive to negative or vice-versa half cycle transition time, and $t_{3}$ depicts the time in which the reciprocating slider rested before moving in the opposite direction.

The characteristics of different time zones changes were based on machine configuration and system properties. Therefore, many experimental test performances focused on a long duration, using a fixed sampling instead of varying sampling rate. Since almost all the tribological experimental process is a slow varying process, the fixed sampling method is widely preferred.

To calculate both the friction coefficients and data representing the static friction, the dynamic friction in the cycles must be extracted (cycle extraction) and segregated from the signal. Therefore, it was done by using eq. 1 .

$Y=\frac{S[\text { signal }-x]}{\left(F_{s} * T_{x}\right)}$

Where, $Y=$ total number of cycles, $\mathrm{S}$ [Signal $-\mathrm{x}]=$ signal data from the sensor (such as friction force, acceleration, among others), $F_{s}=$ sampling frequency, and $T_{x}=$ Time for completion of one cycle (seconds).

Equation 1 was used to extract the total number of cycles. The calculation was performed by pointwise operation. That is, $\mathrm{Y}$ has a separate data sets from the entire cycle. For instance, $\mathrm{S}$ has 120 seconds duration of friction signal data, with the sampling rate of $5 \mathrm{~Hz}$ and period of 2 seconds, then Y will have 12 separate datasets, such as Y1, Y2, Y3... Y12, each will have a signal section equivalent to the duration of 2 seconds and have 10 data points. There are few drawbacks associated with this method, as subsequently discussed.

1) This equation has two assumptions: firstly, the signal data contains an exact number of reciprocating cycles, and the secondly, each cycle has fixed data points. In real-world data, both assumptions hold in a very few cases. For the first assumption, it not necessary for a machine to produce a complete cycle, especially for a heavy duty system. Due to mechanical 
uncertainties, some cycles could be incomplete. For the second assumption, this is related only on the values of $F_{s}$ and $T_{x}$. Although, both values were fixed, it is an accuracy limited to machine's specifications, especially for $T_{x}$ of the reciprocating system. For this present work, the work cycle time was 4 seconds. Despite of been fixed over the time, there were some differences in terms of milliseconds.

2) It was not possible to precisely get the data section of the signal corresponding to one complete cycle, because when the sampling rate was high enough, for example, $20 \mathrm{kHz}$ the error of 10 milliseconds in the completion of one reciprocating cycle resulted to an improper segregation of the cycle and also caused the error to propagate in the following cycles.

3) Another potential errors occurred from the mechanical system's uncertainties and varied the air resistances, electrical noise, material dimension tolerances, machine's start and stop time, among others. The signal data also contained data points corresponding to noises, machine's delayed start (data acquisition started before the machine operation) in addition to the reciprocating cycle.

In long duration tests, all these errors proportionally propagated through the time scale. Therefore, many researchers prefer to use a few random sample cycles from several instances (few 100 cycles from a total of 2500 cycles) for friction calculation instead of all the cycles in the experiment. This further diminished the accuracy of the results obtained. After a random sample of cycles, each cycle was further divided according to Fig. 4 to calculate the static and dynamic friction coefficients. It can be done in 2 ways, one way is to use eq. 1 and $T_{x}$ will be replaced with $t_{1}, t_{2}$, and $t_{3}$, as previously illustrated in Fig. 4, then calculate the average mean. Another method is to calculate according to eq. 2, proposed by Bonny et. al. [23].

$F_{T_{s, \text { stat }}}=\frac{\left|F_{T_{\mathrm{m}} \min }\right|+\left|F_{T_{\mathrm{a}} \max }\right|}{2}$

Where, $F_{T_{\text {s stat }}}=$ static friction coefficient value of the corresponding cycle, $\left|F_{T_{\text {smin }}}\right|=$ minimum absolute peak value of the corresponding cycle, and $\left|F_{T_{a} \max }\right|=$ maximum absolute peak value of the corresponding cycle.

Equation 2 is widely adopted where each cycle $\left(\left|F_{T_{2} \min }\right|\right)$ and $\left|F_{T_{2} \max }\right|$ are used for calculating the static friction coefficient, and the resultant values for all the cycles are appended 
together to produce friction coefficient plot for the entire experimental signal. For the calculation of the dynamic coefficient of friction, eq. 3 was used as similarly adapted from Bonny et .al. [23].

$F_{T, d y n}=\sqrt{\frac{1}{T} * \int_{0}^{T}\left(F_{T}(t)^{2}\right) d t}$

Where, $F_{T_{v} d y n}=$ dynamic friction coefficient value of the corresponding cycle, $F_{T}(t)=$ Cycle's data value at time $t$, and $T=$ time for completion of one cycle.

From eq. 2, it can be concluded that the $\left(F_{T_{i} d y n}\right)$ is the root mean square (RMS) of the data corresponding to a single cycle. There are few drawbacks in the methods mentioned above with respect to calculating static and dynamic friction coefficients, using eqs. 2 and 3. These limitations are thus stated.

1) For the static friction coefficient, the equation only emphasizes absolute maximum and minimum values instead of data spread throughout a specific time, i.e. values under time frame $t_{1}$ and $t_{3}$.

2) For the dynamic friction coefficient, the RMS calculation is a simple measure of the magnitude of the presence in a set of numbers, and it merely illustrates the size of the numbers present in a numerical sequence. In this case, it is the dynamic coefficient of friction, since it occupies a major portion of the cycle. However, this method would be less useful and produce incorrect values when the value of fluctuations is high with the friction coefficient values, due to several factors, such as noise (mechanical and electrical), specimen composition, mechanical behavior (rotating or reciprocating), to mention but a few.

Hence, it is imperative and evident that there is need for a novel technique to overcome these drawbacks

\subsection{Steps followed in the proposed method for friction analysis algorithm}

The process of programming and executing the algorithm was carried out according to the Fig. 3 in the cloud system. The final output was then transferred to the local computer to free up the computing resources in the cloud. Thus, reducing the cost of computing resources. 


\subsubsection{Zero-crossing based on individual cycle extraction}

It is important to segregate the signals in the proper sequence for the better assessment before processing any big data. However, it is not possible to achieve accurate data separated from the outcome of the physical systems during the data acquisition due to several factors, such as noise, among other factors that were earlier mentioned. The large scale tribological setup was connected to many mechanical systems and thus, subjected to mechanical and frictional losses between the components. Been a reciprocating sliding system, the signals recorded had distinctive properties in both positive and the negative half of each cycle. The test was conducted for approximately 3 hours and the recorded data set contained approximately 2700 cycles, including data points related to the noise and disturbance factors. Fig. 5a (i) represents a generic zero-crossing function. This function triggered at events when a periodic signal data points crossed zero. The triggered function was regulated by manually defined hysteresis function, which prevented false trigger due to noise.

The function ensured that the trigger was activated or detected when specific conditions were met for both positive and negative half of the cycle. For a rising edge or a positive part of the cycle, sensing started only when the signal passed below the maximum threshold value minus hysteresis factor value. For a falling edge or a negative part of the cycle, sensing was stopped only when the signal passed above minimum threshold value plus the hysteresis factor value. Fig. 5a (ii) represents a generic implementation of zero-crossing with defined hysteresis, which rejected 3 noises and detected 6 zero-crossing triggers.

Fig. 5b represents one cycle extracted from the friction signal, using the zero-crossing method. Fig . $5 \mathrm{c}$ represents extraction of one cycle from the friction signal using equ. 1. From the comparison made, this concluded that there was a specific error that came from the result, in particular from the mismatch in time and number of data points synchronization, that is the data points in between the timeframe did not represent a complete cycle. The zero-crossing method of cycle extraction was performed on the entire signal data to get data points corresponding to every reciprocating cycle. Then, the period for completion of each cycle was plotted, as shown in Fig. 6 , where $\mathrm{y}$-axis (time) represents the time elapsed for the completion of one cycle and $\mathrm{x}$-axis (no of cycles) represents the corresponding cycle's index number (cycles 1, 2, 3, ., 2700). From Fig. 6, it was evident that with the fixed stroke length and velocity of the reciprocating system, 
the cycle time did not remain consistent. However, this can be neglected as a margin of error when for a heavy-duty system when calculating friction coefficient. Its effects amplified when random cycle sample was used for analysis and resulted to other difficulties in the future analysis of stick-slip behaviors.

Since the zero-crossing method does not rely on the average periodic time for splitting signal data for extraction of all cycles, the drawbacks mentioned previously was eliminated. Moreover, all the extracted cycles were stored in a 2-dimensional dataset with the cycle index number as the first dimension values and its associated data points as the value of the second dimension. Therefore, multiples of each cycle were accessed randomly or sequentially with its index number.

\subsubsection{Method of directed sorting of data points based on the magnitude}

After the separation of each cycle from the whole test signal data, each cycle's data points at different time zones, as illustrated in the Fig. 4 pertaining to static and dynamic friction values needed segregation. As mentioned previously, the existing methods have drawbacks; therefore, to mitigate those drawbacks, a new approach was used. As each cycle can be accessed easily, it was used in the following eq. 4; this has been extended to the parallel processing computing, such as general-purpose computing on graphics processing units (GPGPU) [24], which enabled a significant reduction in computing time.

$\tau_{z}=$ Ascending direction sorting of $\left|C_{x}\right|$

Where, $\left|C_{x}\right|=$ absolute values of the selected cycle with the index $\mathrm{x}, \tau_{z}=$ the output result of the cycle's data points sorted in the ascending direction of its magnitude, and $\mathrm{x}=\mathrm{z}=$ range from 1 to maximum number of cycles.

The result $\left(\tau_{z}\right)$ plot of a cycle with the representation of different time zones is shown in Fig. 7. After this process; it was possible to split and segregate data points corresponding to the static and dynamic friction coefficients and to eliminate the unnecessary data points in the cycle.

\subsubsection{Static friction coefficient}


Static friction is a measure of friction coefficient data points over a few milliseconds from the moment at which the slider starts to move. This friction coefficient was higher in amplitude as compared to the dynamic friction coefficient. The friction coefficient $(\mu)$ data point values of the cycle corresponding to the time zones $t_{1}, t_{2}$ and $t_{3}$ were used with the proposed eq. 5 to calculate the static coefficient of friction.

$\left[\mu_{\text {static }}\right]_{k}=\lim _{k=1 \rightarrow n}\left(\frac{\sum \tau_{k}-\left(\left|F_{t 2}\right|_{k}+\left|F_{t 3}\right|_{k}\right)}{t 2_{k} * F_{s}}\right)$

Where, $\left[\mu_{\text {static }}\right]_{k}=$ static friction coefficient value for the $\mathrm{k}^{\text {th }}$ index cycle, $\tau_{k}=$ result of direction sorting for the $\mathrm{k}^{\text {th }}$ index cycle, $\lim _{k=1 \rightarrow n}=$ represents accessing of cycles from the start to end, $\mathrm{n}=$ total number of cycles in the test data, and $F_{s}=$ sampling frequency $(20 \mathrm{kHz})$.

$\left[\mu_{\text {static }}\right]$, is the data set of static friction coefficient of all the cycles indexed similar to the previously mentioned method of zero-crossing. Therefore, multiples of each cycle data were accessed randomly or sequentially with its index number.

For an experimental test, the total static friction plot was obtained from the appending static friction coefficient sets of all the cycles chronologically. Fig. 8 shows the comparison of the static friction plot between the proposed method and the existing method.

\subsubsection{Dynamic friction coefficient}

Dynamic friction is a measure of friction coefficient data points after the end of the static friction; up to the time at which the slider moves to the rest position, in case of a reciprocating friction system. This friction coefficient was lower in amplitude as compared to the static friction coefficient. The friction coefficient $(\mu)$ values corresponding to the time zones $t_{1}, t_{2}$ and $t_{3}$ were used along with the eq. 6 to calculate the dynamic coefficient of friction.

$\left[\mu_{\text {dynamic }}\right]_{l}=\lim _{l=1 \rightarrow n}\left(\frac{\sum \tau_{l}-\left(\left|F_{t 1}\right|_{l}+\left|F_{t 3}\right|_{l}\right)}{t 1_{l} * F_{s}}\right)$

Where, $\left[\mu_{\text {dynamie }}\right]_{l}=$ static friction coefficient value for the $1^{\text {st }}$ index cycle, $\tau_{l}=$ result of direction sorting for the $1^{\text {th }}$ index cycle, $\lim _{l=1 \rightarrow n}=$ accessing cycles from the start to end, $n=$ total number of cycles in the test data, $F_{s}=$ sampling frequency $(20 \mathrm{kHz}) .\left[\mu_{\text {dynamic }}\right]$ is the data set of static friction coefficient of all the cycles indexed similar to the static friction coefficient. 
Therefore, multiples of each cycle data were accessed randomly or sequentially with its index number. The dynamic friction plot is shown in Fig. 9, and the rest of the process was repeated for a static friction.

\subsection{Statistical analysis of signal for relations of friction coefficients}

In order to validate the proposed method for calculating friction coefficients, a comparison of the magnitude values of the results obtained with the magnitude values of the cycles was conducted. It was impossible to manually compare every individual point due to the large data set obtained. Therefore, this work used statistical methods of spread measurement and one-dimensional analysis of variance (1D-ANOVA) for validation and further analysis.

\subsubsection{Spread calculation on the dynamic coefficient of friction}

A mathematical tool (spread calculation) was used to compute the amount of data spread/distributed relative to the amplitude based on the interquartile range with upper and lower quartile value represented in the Fig. 10. Although, this method was highly specific to the type of data distribution inside the signal and this experimental work signal satisfied certain conditions. Hence, the spread calculation method was used. For each cycle in a test, the spread was plotted, as shown in Fig. 11. The result obtained was very similar to that of the dynamic coefficient of friction. More than an approximately $85 \%$ of the data points belonged to the dynamic coefficient of friction of a cycle.

\subsubsection{One dimensional ANOVA on friction signal friction}

The 1D- ANOVA mathematical tool was used to analyze the behaviors of peaks and valleys occurred at different periods in the friction region. Thus, it illustrated the possibility of in-depth analysis on the friction signal cycles having more accuracies than existing methods. The data point array of a periodic cycle was constructed to be similar to the observations made at different levels of some factors and compute 1D-ANOVA. This was basically a method of categorizing data. For example, it was similar to Table 2 when categorizing data set of materials based on their tensile strengths. 
In ANOVA, observation is a process of classifying the experimental signal based on the defined levels. In this work, 4 levels of a cycle whose data points were sorted in ascending order of the test, as shown in Fig. 12 was adopted. Each level was classified based on the transient time, dynamic friction time, static friction time and slider rest time, as shown in Table 3.

\subsubsection{The theoretical background of ANOVA analysis on friction signal}

The proposed procedure of 1D-ANOVA calculation on friction signal expressed the experimental data corresponding to a cycle. This was necessary to perform the ANOVA on the friction test signal. A summation of 3 parts was carried out, as expressed in eq. 7.

$X_{i m}=\mu_{o}+a_{i}+E_{i m}$

Where, $X_{i m}=m_{t h}$ observation from the $i_{\text {th }}$ level, $\mu_{o}=$ overall mean, $a_{i}=i_{t h}$ level of the classification (ANOVA levels 0, 1,2 and 3), and $E_{i m}=$ random fluctuation.

In ANOVA computation, there were 3 considered properties: ANOVA hypothesis, ANOVA assumptions, ANOVA general method. ANOVA hypothesis, also referred to as the null hypothesis, stating that no level affects the experimental outcome and then looks for evidence to the contrary. ANOVA assumptions states that the data of measurements at each level are normally distributed with mean $\mu_{i}$ and variance $\sigma^{2}$, and have an assumption that $a_{i}$ sum to zero. Finally, the assumption that for each $i$ and $m, E_{i m}$ is normally distributed with mean 0 and variance $\sigma^{2}$. Though, a dynamic friction coefficient part was not exactly a normally distributed quantity in this work. This assumption was compensated by assuming that the distribution was almost high flat at that region, since it occupied about $85 \%$ of the total data.

$T S S=S S A+S S E$

From the ANOVA expressions (eqs. 9-13:

$X_{i m}=m_{t h}$ observation made at the $i_{t h}$ level for $m=0,1, \ldots, \mathrm{n}_{\mathrm{i}}-1$ and $i=0,1, \ldots, \mathrm{k}-1$.

Where, $n_{i}$ is the number of observations at $i_{t h}$ level and $\mathrm{k}$ represents the number of levels. 


$$
\begin{aligned}
& X_{i}=\sum_{i=0}^{k=1} \sum_{m=0}^{n_{i}-1} X_{i m} \\
& \mathrm{n}=\sum_{\mathrm{i}=0}^{k-1} \mathrm{n}_{\mathrm{i}}
\end{aligned}
$$

$\mathrm{SSA}=\sum_{\mathrm{i}=0}^{\mathrm{k}-1}\left(\frac{\mathrm{X}_{\mathrm{i}}^{2}}{\mathrm{n}_{\mathrm{i}}}\right)$

$\mathrm{MSA}=\frac{\mathrm{SSA}}{\mathrm{k}-1}$

$\mathrm{SSE}=\sum_{\mathrm{i}=0}^{\mathrm{k}=1} \sum_{\mathrm{m}=0}^{\mathrm{n}_{\mathrm{i}}-1} \mathrm{x}_{\mathrm{im}}{ }^{2}$

Where, $\mathrm{TSS}=$ total sum of squares, $\mathrm{SSA}=$ measure of variation related to category levels, $\mathrm{SSE}=$ measure of variation related to random fluctuation, and MSA = mean square quantity.

Fig. 13 represents the ANOVA results obtained from the plots with highlighted cycle samples at 6 different time zones, as $S_{1}-S_{6}$, respectively. Fig. 14 represents the friction force signal's sample cycles between these 6 time-frame. Fig. 15 represents the fast Fourier transform for those sample cycles, respectively.

\subsubsection{SSE: Measure of variation related to random fluctuation}

The SSE value for each cycle was computed according to eq. 13; the output of this function reacted proportionally to the rate of change in random noise input data. The random influence noise varied and propagated linearly with time in this work. Change in signal value was regarded as random noise concerning subsequently mentioned cases throughout the test elapsed time.

1) During the initial run, significant changes in dynamic and static friction time zones were regarded as noise, since the material removal was minimum at these instances, caused by minimal material's friction coefficients.

2) More than halfway through experimental test time, the friction coefficients were maximum and constant for some periods. Therefore, changes in dynamic and static frictions were also regarded as noises at these time instances. 
These random noises could be from electrical interferences, machine disturbances, among other sources.

\subsubsection{SSA: Measure of variation related to category levels}

The SSA value for each cycle was computed according to eq. 11. The output of this function responded proportionally to the rate of changes in the percentage of data points occupying the specific timeframe by category levels. For a cycle, the dynamic friction occupied nearly $80 \%$ of the total data points, approximately $15 \%$ of static friction and other $5 \%$ was due to noise, slider rest, among other sources, within this work. Changes in these percentage values were inferred using the SSA and regarded as irrelevant data points.

\subsubsection{MSA: Mean square quantity}

The MSA is calculated according to the eq. 12. The output of this function reacted to a change in amplitude of the friction signal. In other words, a spike or another factor contributed a significant changes in the amplitude, as observed using MSA. These changes occurred at different number of levels or behavior of the levels.

\section{Inference from the computation}

As shown in Fig. 13, the selection of time frame $\left(\mathrm{S}_{1}-\mathrm{S}_{6}\right)$ for the concerned sample cycles was based on the observational changes in the ANOVA results (SSE, SSA and MSA). For example, a sample cycle from the time frame $S_{1}$ was from the instance few minutes after the test system started, i.e. the time at which the influence of friction behavior would be minimum. Both Figs. $14\left(\mathrm{~S}_{1}\right)$ and $15\left(\mathrm{~S}_{1}\right)$ confirmed this phenomenon, where the sample cycle plot in the region of $S_{1}$ and its associated FFT frequency plot showed that there was no other associated frequency components other than the cycle's reciprocating operation frequency and its harmonics.

Moreover, this was similar for the $S_{2}, S_{3}$ and $S_{4}$ samples (Fig. 14), which was taken at the time instances, when the influence of friction behavior started to occur. Therefore, data points in these sample cycles having mutually exclusive frequencies were regarded as noise (i). It is necessary to differentiate and prove that the different frequency components correspond to the noise or the useful signal. It was done by analyzing the frequencies (Fig. 15) persisted throughout the tests (ii) and frequencies originated at the random time (iii). The process was 
done similarly to that of the previous samples $\left(\mathrm{S}_{2}, \mathrm{~S}_{3}\right.$ and $\left.\mathrm{S}_{4}\right)$ by observing changes in the ANOVA results of the sample cycle from time frame $S_{5}$ and $S_{6}$. From Figs. 14 and 15, it can be concluded that both samples were almost identical in terms of time and frequency, based on the ANOVA results obtained. Furthermore, the highlighted area of interest on Fig. 13, between which the sample cycles was taken, i.e. the time frame at which the influence of friction was much higher than the noises.

Where,

(1) Frequencies $=\sim 300 \mathrm{~Hz}$ and $\sim 340 \mathrm{~Hz}$ (i).

(2) Frequencies $=\sim 220 \mathrm{~Hz}$ and $\sim 280 \mathrm{~Hz}$ (ii).

(3) Frequencies $=\sim 50 \mathrm{~Hz}, \sim 100 \mathrm{~Hz}$ and $\sim 150 \mathrm{~Hz}$ (iii).

\section{Conclusion}

Static and dynamic friction coefficients for material have been determined using different methodologies: zero-crossing detection and a new algorithm. These novel techniques mitigated some of the drawbacks of the existing methods, such as random sampling method. The ANOVA computation on the friction signal also emphasized that the use of a randomly sampled signal on a long time experimental test resulted to an erroneous values. By comparing different ANOVA results with specific time frames, different frequency components were identified and differentiated. This was beneficial as the noise parts of the signal were detected. The identification of noise portions are quite difficult with the conventional methods. By incorporating different signal processing methodologies, such as time, frequency and timefrequency domain analysis, it was possible to analyze the in-depth behavior of the stick-slip phenomena of the friction system. Improved implementation of the proposed computing methods would facilitate real-time computing and compensation on the friction related application systems, such as automotive braking system. Consequently, it would be possible to use the

braking system in a pulsed switching manner to achieve improved performance in the static and dynamic friction regions. 


\section{References}

[1] Feeny B, Guran A, Hinrichs N, Popp K. A historical review on dry friction and stick-slip phenomena. Appl Mech Rev 1998;51:321-41. doi:10.1115/1.3099008.

[2] Gao C, Kuhlmann-Wilsdorf D, Makel DD. Fundamentals of stick-slip. Wear 1993;162164:1139-49. doi:10.1016/0043-1648(93)90133-7.

[3] Awrejcewicz J, Olejnik P. Occurrence of stick-slip phenomenon. J Theor Appl Mech 2007;45:33-40.

[4] Pratt TK, Williams R. Non-linear analysis of stick/slip motion. J Sound Vib 1981;74:53142. doi:10.1016/0022-460X(81)90417-X.

[5] Gao C, Kuhlmann-Wilsdorf D, Makel DD. The dynamic analysis of stick-slip motion. Wear 1994;173:1-12. doi:10.1016/0043-1648(94)90251-8.

[6] Real FF, Lobo DM, Ritto TG, Pinto FANC. Experimental analysis of stick-slip in drilling dynamics in a laboratory test-rig. J Pet Sci Eng 2018;170:755-62. doi:10.1016/j.petrol.2018.07.008.

[7] Dong C, Yuan C, Bai X, Qin H, Yan X. Investigating relationship between deformation behaviours and stick-slip phenomena of polymer material. Wear 2017;376-377:1333-8. doi:10.1016/j.wear.2017.01.061.

[8] Viswanathan K, Sundaram NK. Distinct stick-slip modes in adhesive polymer interfaces. Wear 2017;376-377:1271-8. doi:10.1016/j.wear.2016.12.017.

[9] Renaud F, Chevallier G, Dion JL, Taudire G. Motion capture of a pad measured with accelerometers during squeal noise in a real brake system. Mech Syst Signal Process 2012;33:155-66. doi:10.1016/j.ymssp.2012.06.027.

[10] Thomsen JJ, Fidlin A. Analytical approximations for stick-slip vibration amplitudes. Int J Non Linear Mech 2003;38:389-403. doi:10.1016/S0020-7462(01)00073-7.

[11] Lima R, Sampaio R. Construction of a statistical model for the dynamics of a base-driven stick-slip oscillator. Mech Syst Signal Process 2017;91:157-66. doi:10.1016/j.ymssp.2016.12.038.

[12] Bhattacharyya P, Sengupta D, Mukhopadhyay S. Cutting force-based real-time estimation of tool wear in face milling using a combination of signal processing techniques. Mech Syst Signal Process 2007;21:2665-83. doi:10.1016/j.ymssp.2007.01.004.

[13] Kong D, Chen Y, Li N. Gaussian process regression for tool wear prediction. Mech Syst Signal Process 2018;104:556-74. doi:10.1016/j.ymssp.2017.11.021.

[14] Neis P, Ferreira N, Matoso L, Masotti D, et al. "Definition of a Suitable Parameter for Characterizing Creep-Groan Propensity in Brake Pads," SAE Technical Paper 2015-36-0004, 2015, https://doi.org/10.4271/2015-36-0004.

[15] Neis PD, Ferreira NF, Poletto JC, Matozo LT, Masotti D. Quantification of brake creep groan in vehicle tests and its relation with stick-slip obtained in laboratory tests. J Sound Vib 2016;369:6376. 
[16] Masotti D, Neis P, Ferreira N, Gomes K. et al. "Experimental Evaluation of Surface Morphology Characteristics During Stick-Slip Process at Low Speed Sliding Test," SAE Technical Paper 201501-2685, 2015, https://doi.org/10.4271/2015-01-2685.

[17] Martinez K, Hart JK, Basford PJ, Bragg GM, Ward T, Young DS. A geophone wireless sensor network for investigating glacier stick-slip motion. Comput Geosci 2017;105:103-12. doi:10.1016/j.cageo.2017.05.005.

[18] Dimla DE. Sensor signals for tool-wear monitoring in metal cutting operations - a review of methods. Int J Mach Tools Manuf 2000;40:1073-98.

[19] Boud F, Gindy NNZ. Application of multi-sensor signals for monitoring tool/workpiece condition in broaching. Int J Comput Integ Manuf 2008;21:715-29. doi:10.1080/09511920701233357.

[20] Chung KT, Geddam A. A multi-sensor approach to the monitoring of end milling operations. 2003;139:15-20. doi:10.1016/S0924-0136(03)00175-4.

[21] Segreto T, Simeone A, Teti R. Multiple sensor monitoring in nickel alloy turning for tool wear assessment via sensor fusion. Procedia CIRP 2013;12:85-90. doi:10.1016/j.procir.2013.09.016.

[22] Subramanian K, Nagarajan R, De Baets P, Subramaniam S, Thangiah W, Sukumaran J. Ecofriendly mono-layered PTFE blended polymer composites for dry sliding tribo - systems. Tribol Int 2016;102:569-79. doi:https://doi.org/10.1016/j.triboint.2016.06.015.

[23] Bonny K, De Baets P, Vleugels J, Huang S, Lauwers B. Dry reciprocating sliding friction and wear response of WC-Ni cemented carbides. Tribol Lett 2008;31:199-209. doi:10.1007/s11249008-9352-z.

[24] Oancea B, Tudorel A, Mariana DR, “GPGPU computing”’ arXiv:1408.6923, 2012. 
Fig. 1. Fabricated BFPTFEC laminate, using hand layup technique (i) schematic view (ii) side view.

Fig. 2. Schematic diagram of experimental setup used for friction and wear measurements.

Fig. 3. Algorithm flow for computing static and dynamic friction coefficients.

Fig. 4. Friction curve plot for reciprocating sliding piston.

Fig. 5a (i). Generic zero-crossing function.

Fig. 5a (ii). Generic zero-crossing with defined hysteresis.

Fig. 5b. Comparison between cycle extraction on (i) proposed and (ii) conventional methods.

Fig. 6. A completion time of each cycle.

Fig. 7. Result of a cycle's data points sorted in the ascending direction of its magnitude.

Fig. 8. Comparison of static coefficient of friction curve plots between two methods.

Fig. 9. Comparison of dynamic coefficient of friction curve plots between two methods.

Fig. 10. Spread computed result for the test data.

Fig. 11. Interquartile range (IQR) analogy (Image courtesy: Centers for disease control and prevention, USA).

Fig. 12. ANOVA level classification.

Fig. 13. ANOVA results for the test signal.

Fig. 14. Sample friction cycles at time frames of $S_{1}-S_{6}$.

Fig. 15. FFT sample cycle at time frames of $S_{1}-S_{6}$. 


\section{Table 1}

Specifications of wear test

\begin{tabular}{ll}
\hline Property & Value \\
\hline Type of test platform & Linear reciprocating \\
Operating type & Uniform, sinusoidal \\
Normal load & $10 \mathrm{kN}$ \\
Friction force & $100 \mathrm{kN}$ \\
Velocity & $50 \mathrm{~mm} / \mathrm{S}$ \\
Time frame for each cycle & $4 \mathrm{~seconds}$ \\
Total displacement per cycle & $200 \mathrm{~mm}$ \\
Stroke length per half cycle & $100 \mathrm{~mm}$ \\
Total operation time & $\sim 3 \mathrm{hours}$ \\
Total number of cycles & $\sim 2700$ \\
Specimen material dimension (area and thickness) & $2500 \mathrm{~mm}{ }^{2} \mathrm{x} 7 \mathrm{~mm}$ \\
\hline
\end{tabular}

\section{Table 2}

Example of ANOVA level classifications

\begin{tabular}{ccc}
\hline No. of levels & Material types & *Yield strength (MPa) \\
\hline Level 0 & Wood & 69 \\
Level 1 & Light metal & 270 \\
Level 2 & Heavy metal & 1000 \\
Level 3 & Very heavy metal & 2500
\end{tabular}

*Strength values was tabulated for example purposes only and does not implies to real-world material properties. 
Table 3

ANOVA level classification for the test signal

\begin{tabular}{cl}
\hline No. of levels & Categories based on time zone range \\
Level 0 & Cycle transition time \\
Level 1 & Dynamic coefficient friction time \\
Level 3 & Static coefficient friction time \\
\end{tabular}

